\title{
Erratum
}

\section{On eigenvalue pinching in positive curvature}

\section{Peter Petersen ${ }^{\star}$}

Department of Mathematics, University of California, Los Angeles, CA 90095-1555, USA (e-mail: petersen@math.ucla.edu)

Invent. math. 138, 1-21 (1999)

Oblatum 2-V-2003 \& 23-VI-2003

Published online: 15 September 2003 - C Springer-Verlag 2003

It has been pointed out that the proof of Lemma 6.2 on p. 17 in [2] contains two errors. The first is that the definition of degree is incorrect. The correct definition is

$$
\operatorname{deg}(\Phi)=\frac{1}{\operatorname{vol} S^{n}} \int_{M} \operatorname{det}(d \Phi) .
$$

Since $\operatorname{vol} M \leq \operatorname{vol} S^{n}$ this does not affect the arguments below.

The other more serious mistake is that the set $A_{\delta}$ need not be connected so we cannot assume that $\operatorname{det}(d \Phi)$ has constant sign on this set. This means that the proof of Lemma 6.2 is incomplete as it stands. The author does not see a way of correcting this particular error nor does he know of an alternate proof which is simpler than what is already in the literature. The author would like to mention that Cheeger has written an article [1], which contains a complete and streamlined account on convergence of manifolds with Ricci curvature bounds.

Note that this does not affect Theorem 1.1 of [2]. This result is proven in Sects. 3, 4, 5 and does not depend on Sect. 6.

\section{References}

1. Cheeger, J.: Degeneration of Riemannian Metrics under Ricci Curvature Bounds. Scuola Normale Superiore Publications, Lezioni Fermiane 12

2. Petersen, P.: On eigenvalue pinching in positive Ricci curvature. Invent. Math. 138, 1-21 (1999)

* Supported in part by the NSF

Mathematics Subject Classification (1991): 53C20 